\title{
Correction to: Multivariate Gaussian and Student- $t$ process regression for multi-output prediction
}

\author{
Zexun Chen ${ }^{1,2}$ (1) $\cdot$ Bo Wang ${ }^{1} \cdot$ Alexander N. Gorban ${ }^{1}$
}

Published online: 27 February 2020

(C) Springer-Verlag London Ltd., part of Springer Nature 2020

\section{Correction to: Neural Computing and Applications}

https://doi.org/10.1007/s00521-019-04687-8

Unfortunately, Theorem 3 was published with incorrect equations in the online publication of the article.

The correct theorem should read as follows:

Theorem 3 (Marginalization and conditional distribution) Let $X \sim \mathcal{M N}_{n, d}(M, \Sigma, \Omega)$ and partition $X, M, \Sigma$ and $\Omega$ as
2. $X_{1 c} \sim \mathcal{M N}_{n, d_{1}}\left(M_{1 c}, \Sigma, \Omega_{11}\right)$, $X_{2 c} \mid X_{1 c} \sim \mathcal{M N}_{n, d_{2}}\left(M_{2 c}+\left(X_{1 c}-M_{1 c}\right) \Omega_{11}^{-1} \Omega_{12}, \Sigma, \Omega_{22 \cdot 1}\right)$, where $\Sigma_{22 \cdot 1}$ and $\Omega_{22 \cdot 1}$ are the Schur complement [30] of $\Sigma_{11}$ and $\Omega_{11}$, respectively,

$\Sigma_{22 \cdot 1}=\Sigma_{22}-\Sigma_{21} \Sigma_{11}^{-1} \Sigma_{12}, \quad \Omega_{22 \cdot 1}=\Omega_{22}-\Omega_{21} \Omega_{11}^{-1} \Omega_{12}$.

$$
\begin{aligned}
& \left.X=\left[\begin{array}{c}
X_{1 r} \\
X_{2 r}
\end{array}\right] \begin{array}{c}
n_{1} \\
n_{2}
\end{array}=\left[\begin{array}{cc}
X_{1 c} & X_{2 c} \\
d_{1} & d_{2}
\end{array}\right], \quad M=\left[\begin{array}{c}
M_{1 r} \\
M_{2 r}
\end{array}\right] \begin{array}{c}
n_{1} \\
n_{2}
\end{array}=\begin{array}{cc}
M_{1 c} & M_{2 c}
\end{array}\right] \\
& \Sigma=\begin{array}{cc}
{\left[\begin{array}{cc}
\Sigma_{11} & \Sigma_{12} \\
\Sigma_{21} & \Sigma_{22}
\end{array}\right]} & n_{1} \\
n_{2} \\
n_{1} & n_{2}
\end{array} \quad \text { and } \quad \Omega=\left[\begin{array}{cc}
\Omega_{11} & \Omega_{12} \\
\Omega_{21} & \Omega_{22}
\end{array}\right] \begin{array}{c}
d_{1} \\
d_{1}
\end{array}
\end{aligned}
$$

where $n_{1}, n_{2}, d_{1}, d_{2}$ is the column or row length of the corresponding vector or matrix. Then,

1. $X_{1 r} \sim \mathcal{M N}_{n_{1}, d}\left(M_{1 r}, \Sigma_{11}, \Omega\right)$,

$$
X_{2 r} \mid X_{1 r} \sim \mathcal{M N}_{n_{2}, d}\left(M_{2 r}+\Sigma_{21} \Sigma_{11}^{-1}\left(X_{1 r}-M_{1 r}\right), \Sigma_{22 \cdot 1}, \Omega\right) \text {; }
$$

The original article has been updated accordingly.

Publisher's Note Springer Nature remains neutral with regard to jurisdictional claims in published maps and institutional affiliations.

The original article can be found online at https:// doi.org/10.1007/s00521-019-04687-8.

\section{Zexun Chen}

z.chen3@exeter.ac.uk; sxtpy2010@gmail.com

1 Department of Mathematics, University of Leicester,

Leicester LE1 7RH, UK

2 Present Address: College of Engineering, Mathematics and Physical Sciences, University of Exeter, Exeter EX4 4QF, UK 